\title{
The burden of Candida species colonization in NICU patients: a colonization surveillance study
}

\author{
Corresponding author \\ Daniela Maria Geraci \\ Department of Sciences for Health Promotion and Maternal-Infant Care "G. D'Alessandro," Hygiene Section \\ University of Palermo \\ Via del Vespro 133, CAP 90127, Palermo, Italy \\ Phone : $00390916553624 / 00393287866378$, \\ E-mail: danielamaria.geraci@unipa.it / geracidaniela@yahoo.it
}

GERACI DM, VIRGA A, VECCHIO D, GRAZIANO G, SAPORITO L, INSINGA V, MAIDA CM, MAMMINA C, GIUFFRE M

Department of Sciences for Health Promotion and Maternal- Infant Care "G. D'Alessandro," Hygiene Section, University of Palermo, Palermo, Italy

\section{ABSTRACT}

Fungal infections are an important cause of morbidity and mortality in neonatal intensive care units (NICUs). The identification of specific risk factors supports prevention of candidemia in neonates. Effective prophylactic strategies have recently become available, but the identification and adequate management of high-risk infants is still a priority. Prior colonization is a key risk factor for candidemia. For this reason, surveillance studies to monitor incidence, species distribution, and antifungal susceptibility profiles, are mandatory. Among 520 infants admitted to our NICU between January 2013 and December 2014, 472 (90.77\%) were included in the study. Forty-eight out of 472 (10.17\%) patients tested positive for Candida spp. (C.), at least on one occasion. All the colonized patients tested positive for the rectal swab, whereas 7 patients also tested positive for the nasal swab. Fifteen out of 472 patients (3.18\%) had more than one positive rectal or nasal swab during their NICU stay. Moreover, 9 out of 15 patients tested negative at the first sampling, suggesting they acquired Candida spp. during their stay. Twenty-five of forty-eight (52.1\%) colonized patients carried C.albicans and 15/48 (31.25\%) C.parapsilosis. We identified as risk factors for Candida spp. colonization: antibiotic therapy, parenteral nutrition, the use of a central venous catheter, and nasogastric tube. Our experience suggests that effective microbiological surveillance can allow for implementing proper, effective and timely control measures in a highrisk setting.

Key words: Candida, surveillance, NICU

\section{INTRODUCTION}

Fungal infections are an important cause of increased morbidity and mortality in infants admitted to neonatal intensive care units (NICUs). In very low birth weight (VLBW) infants Candida (C.) albicans is the third most common cause of neonatal late onset sepsis (LOS). (1) The overall incidence of candidemia in NICUs is increasing because of longer survival and more invasive procedures related with the intensive care of extremely preterm infants. According to a multicentric study carried out in Virginia, the rate of nosocomial bloodstream infection due to Candida species in babies admitted to NICU is reported as 12.3/1,000 admissions and $0.64 / 1,000$ patient days. (2) Many factors account for the high risk of systemic fungal infections (SFI) in preterm infants in the NICU. They include: prior antibiotic exposure, hyper alimentation, use of intravenous fat emulsions and endotracheal intubation. $(3,4)$ Prior colonization is a key risk factor for candidemia and it has been identified as the first step in pathogenesis of invasive candidiasis. (5) Colonization of the infant occurs early in life and this is affected by a variety of common practices in the NICU. About $60 \%$ of VLBW neonates are colonized during their first month in the NICU, (6) with a peak incidence during the 2nd and 3rd week of life. About $85 \%$ of them acquire the fungus horizontally (nosocomial acquisition) vs. 15\% who are colonized vertically (i.e. from the mother). It is very difficult to reconstruct transmission on the ward. (8)

The interaction between colonization and risk factors has been investigated experimentally and clinically. Bendel et al. (9) have shown that dissemination takes place when dexamethasone is given to rats treated with antibiotics and colonized by fungi. A specific role for hyperglycaemia, colonization of the CVC (central venous catheter), number of colonized sites and candiduria, in determining the progression to dissemination and SFI has been shown as well. (4)

Colonization of neonates with Candida in the NICU is a problem that requires attention, especially in patients with identified risk factors. A similar approach is needed as with other dangerous colonizations, such as Meticillin Resistan Staphylococcus Aureus. $(10,11)$ In fact, the combination of "nearly endemic" presence of Candida colonization in neonates with other risks factors, increases the risk of SFI. Prevention of candidemia in neonates is supported by identification and adequate management of specific risk factors, including low birth weight, use of invasive devices, prolonged hospitalization and use of broad-spectrum antimicrobial agents. Effective prophylactic strategies have recently become available, but the identification of the best possible strategies to manage high-risk infants is still a priority. Previous experience with the circulation of pathogenic microorganisms in the NICU, suggests that for fungi it is also mandatory to conduct surveillance studies to monitor incidence, species distribution, and antifungal susceptibility profiles. (12)

To assess the epidemiology of Candida spp. colonization in hospitalized neonates in our setting, we performed a prospective cohort study at the tertiary NICU of the University Hospital "Paolo Giaccone", Palermo, Italy.

\section{MATERIALS AND METHODS}

Among 520 infants admitted to the NICU between January 2013 and December 2014, $472(90.77 \%)$ were included in the study. Major clinical characteristics of infants were: malformations (101/472; 
$21.4 \%)$, prematurity $(163 / 466 ; 35 \%)$ and surgical procedures $(21 / 472 ; 4.4 \%)$. Other important part of infants were outborn admissions (195/469; 41.6\%). The NICU includes one intensive care room, consisting of 8 cot spaces, and one intermediate care room, with a further 8 cot spaces. The average nurse to patient ratio is $1: 3$ and $1: 4$ in the two sections, respectively. The NICU ward is open to parents for 2 hours in the morning and 4 hours in the afternoon, so that they can be progressively involved in the general care of their child under the guidance and supervision of staff. Early breastfeeding is supported.

During the study period we collected weekly nasal and rectal swabs from each neonate hospitalized in the NICU. Inclusion criteria were: admission to NICU between January 2013 and December 2014, a hospital stay of at least 48 hours, complete demographic and clinical data and the collection of at least one nasal swab. Moreover, demographic and clinical data expected to affect Candida colonization were recorded for each patient. On admission, demographic characteristics, gestational age, birth weight, born in this or another NICU condition, delivery type, APGAR score and comorbid conditions were recorded. During the NICU stay, qualitative and quantitative data were collected for the following variables: presence of central vascular access devices, endotracheal intubation and mechanical ventilation, nasal continuous positive airway pressure (nCPAP), peripheral catheters, type of feeding (i.e. parenteral nutrition, enteral nutrition with oral suction or gavage, breast milk, formula), need and timing of surgery, antibacterial and antifungal drug therapy, length of stay and survival status at discharge.

Nasal and rectal swabs were inoculated on Sabouraud agar dextrose (Oxoid, Basingstoke, UK) and incubated at $30^{\circ} \mathrm{C}$ for 4 days. The Candida isolates were identified by standard procedures, i.e. morphology on Corn meal agar plates, germ-tube test (GTT) in fetal calf serum, Candida chromogenic agar (ChromAgar Candida, Laboratorios Conda; Madrid, Spain), and biochemical analysis with the API ${ }^{\circledast} 20 \mathrm{C}$ AUX identification system (bioMérieux, Marcy-l'Etoile, France).

Infants were categorized as colonized by Candida spp. when at least one nasal swab tested positive. We evaluated Candida spp. colonization rate and assessed the related risk factors.

All data recording and statistical analyses were performed by EpiInfo (CDC software), and Fisher's exact test was used to assess risk factors, assuming as statistically significant a $\mathrm{p}$ value $<0.05$.

\section{RESULTS}

In this two-year study we enrolled 472 out of 520 patients admitted to the NICU, for which we collected complete microbiological, clinical and demographic data. The main features of the population under study are summarized in Table 1 . Fortyeight out of $472(10.17 \%)$ patients tested positive for Candida spp. at least on one occasion. In particular, 25 patients tested positive for C. albicans, 15 for C. parapsilosis, 6 for C. glabrata, 1 for C. guilliermondi and 1 patient tested positive both for C. albicans and C. parapsilosis. All colonized patients had a positive rectal swab, whereas 7 patients also had a positive nasal swab. Fifteen out of 472 patients $(3.18 \%)$ had more than one positive rectal or nasal swab during their NICU stay. Moreover, 9 out of 15 patients tested negative at the first sampling, suggesting they acquired Candida spp. during their stay.

The reported carriage rate was $8.73 \%(11 / 126)$ in the first semester, $10.74 \%(13 / 121)$ in the second, $8.04 \%$ $(9 / 112)$ in the third and $13.27 \%(15 / 113)$ in the fourth, as shown in Figure 1.

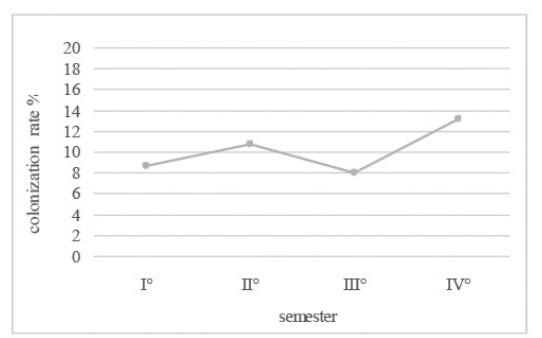

Figure 1. Carriage rate for semesters of study.

Demographic and clinical characteristics of the population under study are summarized in Table 1.

A total of $296 / 472(62.7 \%)$ enrolled patients received at least one antibiotic during their hospitalization and 38/471 $(8.07 \%)$ received at least one antifungal. $36 / 471$ (7.64\%) patients received fluconazole prophylaxis, among these we found 3 colonization cases $(3 / 36,8.33 \%)$. In infants who did not received fluconazole prophylaxis we found 45 colonization cases $(45 / 436,10.32 \%)$. No statistically significant difference was found between these two groups ( $\mathrm{p}$-value $=0.49$ ).

We investigated the association between Candida colonization and clinical and de- mographic data. Table 2 summarizes data about risk factors. Administration of antibiotic therapy, use of a CVC and parenteral nutrition were significantly associated with Candida colonization.

No infection cases of any kind were reported during the study.

\section{DISCUSSION}

The results of this study clearly suggest that effective microbiological surveillance can help maintain the quality of care provided. The microbiological surveillance of Candida spp. colonization allowed us to identify risk factors, to monitor groups of highrisk patients and to implement effective and timely control measures in this high risk setting. The prevalence of colonization by Candida spp. in our NICU is near $10 \%$ and it is lower than other studies reported. $(4,9,13)$ However, we underline that the population involved and the patients enrolled in our study differ in some measure from those of others NICUs. Our patients have a higher gestational age and birth weight (respectively 37.1 weeks and 2795 grams) when compared to other study populations. These demographic data, involving less measures of care by healthcare workers, could partially explain the lower colonization rate. Moreover, no infection cases were reported during the study.

According to prior studies, the rectum is the most common site of colonization. C.albicans and C.parapsilosis are the two most common organisms. (1417) Twenty-five of forty-eight (52.1\%) colonized patients carried C.albicans and 15/48 (31.25\%) C.parapsilosis. The rate of C.parapsilosis colonization is modestly higher than data reported in other paediatric units $(2,18,19)$, so it could be considered an important and distinctive feature of our NICU, requiring deeper analysis.

We identified as risk factors for Candida spp. Colonization: antibiotic therapy, parenteral nutrition, the use of CVCs, and nasogastric tubes. Our data are clearly in agreement with the current knowledge in this field. Saiman et al. (4) reported that the use of third generation cephalosporins was associated with both C.albicans and C.parapsilosis colonization. Moreover, the insertion of central venous catheters or use of intravenous lipids were found to be risk factors for C.albicans.

By contrast, no significant association with gestational age and birth weight was reported in our study. Until now, data we have collected do not indicate a statistically significant association with these variables 
Table 1. Demographic and clinical characteristics of 472 neonates included in the study.

\begin{tabular}{|c|c|}
\hline Variable & \\
\hline Male, No. (\%) & $280 / 472(59,4)$ \\
\hline Caesarean section, No. (\%) & $268 / 472(58,9)$ \\
\hline Born in onother NICU , No. (\%) & $195 / 472(41.6)$ \\
\hline Preterm, No. (\%) & $162 / 472(34.3)$ \\
\hline Malformations, No. (\%) & $101 / 472(21.4)$ \\
\hline Twins, No. (\%) & $41 / 472(9.1)$ \\
\hline Gestational age in weeks, median (IQR) & $38(35-39)$ \\
\hline Birth weight in grams, median (IQR) & $2940(2280-3300)$ \\
\hline 5’ APGAR score <8, No. (\%) & $46 / 472(9.75)$ \\
\hline Days of hospital stay, median (IQR) & $12(9-20)$ \\
\hline Surgical intervention, No. (\%) & $21 / 472(4.4)$ \\
\hline Breastfeeding, No. (\%) & $304 / 472(64.5)$ \\
\hline Formula, No. (\%) & $452 / 472(95.8)$ \\
\hline \multicolumn{2}{|l|}{ Invasive devices } \\
\hline Peripheral catheter, No. (\%) & $366 / 472(77.5)$ \\
\hline Central catheter, No. (\%) & $126 / 471(26.8)$ \\
\hline nCPAP, No. (\%) & $80 / 472(16.9)$ \\
\hline Endotracheal tube, No. (\%) & $57 / 472(12.1)$ \\
\hline Parenteral nutrition, No. (\%) & $354 / 472(75)$ \\
\hline \multicolumn{2}{|l|}{ Antimicrobial therapy } \\
\hline Ampicillin/sulbactam, No, (\%) & $286(60.6)$ \\
\hline Amoxicillin/clavulanic acid, No, (\%) & $17(3.60)$ \\
\hline Aminoglycosides, No, (\%) & $256(54.2)$ \\
\hline Cephalosporins, No, (\%) & $13(2.8)$ \\
\hline Glycopeptides, No, (\%) & $12(2.5)$ \\
\hline Fluconazole, No, (\%) & $36(7.6)$ \\
\hline Micafungin, No, (\%) & $3(0.6)$ \\
\hline Metronidazole, No, (\%) & $2(0.4)$ \\
\hline
\end{tabular}

IQR, interquartile range; nCPAP, nasal continuous positive airway pressure.

Table 2. Risk factors for Candida colonization

\begin{tabular}{|c|c|c|c|c|}
\hline Risk factors & $\begin{array}{l}\text { Colonized } \mathrm{n}=48 \\
\text { No. }(\%)\end{array}$ & $\begin{array}{l}\text { Not colonized } \mathrm{n}=424 \\
\text { No. }(\%)\end{array}$ & $\begin{array}{l}\text { RR } \\
(95 \% \mathrm{CI})\end{array}$ & $\begin{array}{l}\text { P-value } \\
\text { (Fisher exact test) }\end{array}$ \\
\hline Antibiotic therapy & $43(89.6)$ & $253(59.7)$ & $1.14(1.07-1.19)$ & $<0.001^{*}$ \\
\hline Fluconazole prophylaxis & $3(6.2)$ & $33(7.8)$ & $0.98(0.88-1.08)$ & 0.49 \\
\hline CVC & $23(47.9)$ & $103(24.3)$ & $1.13(1.04-1.24)$ & $<0.001^{\star}$ \\
\hline Parenteral nutrition & $43(89.6)$ & $311(73.3)$ & $1.09(1.03-1.15)$ & $<0.001^{\star}$ \\
\hline Nasogastric tube & $19(39.6)$ & $114(26.9)$ & $1.07(0.98-1.15)$ & 0.15 \\
\hline Born in onother NICU & $21(47.7)$ & $173(41.1)$ & $0.98(0.93-1.05)$ & 0.37 \\
\hline Male gender & $30(59)$ & $249(62.5)$ & $1.01(0.95-1.08)$ & 0.38 \\
\hline $\begin{array}{l}\text { Gestational age, weeks } \\
\text { Median (IQR) }\end{array}$ & $38(36-39)$ & 38 & Not suitable & 0.50 \\
\hline Caesarean section & $28(59.6)$ & $239(58.8)$ & $1.01(0.94-1.07)$ & 0.52 \\
\hline $\begin{array}{l}\text { Birth weight, grams } \\
\text { Median (IQR) }\end{array}$ & $3070(2110-3470)$ & $2910(2290-3290)$ & Not suitable & 0.55 \\
\hline
\end{tabular}

CVC, central venous catheter; CI, 95\% confidence interval; IQR, interquartile range; RR, Relative risk; $95 \%{ }^{*}=$ significant p-value 
( $\mathrm{p}$-value $\geq 0.05$ ). Most likely this data is related to inherent features of our population and to the limited numbers of cases.

Antifungal prophylaxis with fluconazole proved to be useful in the management of fungal colonization and infections in adult immunocompromised patients and effective in terms of reduction of candidemia rates. For these reasons it has been introduced in the routine prevention of invasive fungal diseases in other clinical contexts such as neonatology, targeted to preterm infants characterized by an immature immune system. (20-22)

In our study, $7.64 \%$ of enrolled patients received fluconazole prophylactically. (17) There was no statistically significant difference between the treated and not treated groups in terms of colonization rate. This data could be partially related to the low number of treated patients in our study population and it emphasises the need for a continuous surveillance study. We underline that this study is still in place at our NICU. It is our intent to carry on a similar analysis in the next few years to more precisely asses the connection with all these risk factors.

Our understanding of the epidemiology of Candida spp. has grown appreciably over the past years. (19) In this context, we underline the effectiveness of a microbiological surveillance plan. It is important to define the local epidemiology and to monitor rapid changes in the rates of infection, potential risk factors, and emerging pathogen species. Continuous and close surveillance plays a key role in the management of high-risk wards and it could support the development of rational prevention and control measures to achieve better quality of care.

\section{ACKNOWLEDGEMENTS}

This manuscript presents data from a surveillance study still in progress. This may be viewed as a limitation but it is also a motivation for improvement of the study. Additional and more detailed data will be available in the future. We would like to thank all the personnel of the NICU for their cooperation and support.

\section{REFERENCES}

1. Costa-de-Oliveira S, Pina-Vaz C, Mendonça D, Rodrigues AG. A first Portuguese epidemiological survey of fungaemia in a university hospital. Eur J Clin Microbio 2008;127:365-74.

2. Rangel-Frausto MS, Wiblin T, Blumberg HM, Saiman L, Patterson J, Rinaldi M, Wenzel RP. National epidemiology of mycoses survey (NEMIS): variations in rates of bloodstream infections due to Candida species in seven surgical intensive care units and six neonatal intensive care units. Clin Infect Dis 1999;29.2:253-8.

3. Weese-mayer DE, Fondriest DW, Brouillette RT, Shulman ST. Risk factors associated with candidemia in the neonatal intensive care unit: a case-control study. Pediatr Infect Dis J 1987;6.2:190-6.

4. Saiman L, Ludington E, Pfaller M, Rangel-Frausto S, Wiblin RT, Dawson J, Wenzel RP. Risk factors for candidemia in neonatal intensive care unit patients. Pediatr Infect Dis J 2000;19.4:319-24.

5. Cole GT, Halaiva AA, Anaissie E. The role of the gastrointestinal tract in hematogenous candidiasis: from the laboratory to the bedside. Clin Infect Dis 1996; 22(suppl 2):S73-88.

6. Kicklighter SD, Springer SC, Cox T, Hulsey TC, Turner RB. Fluconazole for prophylaxis against candidal rectal colonization in the very low birth weight infant. Pediatrics 2001;107.2:293-8.

7. Kaufman DA. Fluconazole prophylaxis: can we eliminate invasive Candida infections in the neonatal ICU? Curr Opin Pediatr 2008;20.3:332-40.

8. Kumar ARUN, Yadav APARNA, Gathwala GEETA, Gagneja DEEP, Chaudhary U, Gill PS, Gagneja D. Study of risk factors for candida species colonisation of neonatal intensive care unit patient. Int J Pharm Bio Sci 2012;3:193-9.

9. Bendel CM. Colonization and epithelial adhesion in the pathogenesis of neonatal candidiasis. Semin Perinatol 2003;27.5:357-64.

10. Giuffrè M, Amodio E, Bonura C, Geraci DM, Saporito L, Ortolano R, et al. Methicillin-resistant Staphylococcus aureus nasal colonization in a level III neonatal intensive care unit: Incidence and risk factors. Am J Infect Contr 2015;43(5):476-81.

11. Geraci DM, Giuffrè M, Bonura C, Matranga D, Aleo A, Saporito L, et al. Methicillin-resistant Staphylococcus aureus colonization: A three-year prospective study in a neonatal intensive care unit in Italy. PLoS ONE 2014;9(2):e87760.

12. Giuffre M, Geraci DM, Bonura C, Saporito L, Graziano G, Insinga V, et al. The increasing challenge of multidrug-resistant gram-negative bacilli: results of a 5-year active surveillance program in a neonatal intensive care unit. Medicine (United States) 2016;95(10): e3016.

13. Huang YC, Li CC, Lin TY, Lien RI, Chou YH, Wu JL, Hsueh C. Association of fungal colonization and invasive disease in very low birth weight infants. Pediatr Infect Dis J 1998;17.9:819-22.

14. Baley JE, Kliegman RM, Boxerbaum B. Fungal colonization in the very low birth weight infant. Pediatrics 1986;78:225-32.

15. El-Mohandes AE, Johnson-Robbins L, Keiser JF, et al. Incidence of Candida parapsilosis, colonization in an intensive care nursery population and its association with invasive fungal disease. Pediatr Infect Dis J 1994;13:520 — 524.

16. Kicklighter SD, Springer SC, Cox T, HulseyTC, Turner RB. Fluconazole for prophylaxis against candidal rectal colonization in the very low birth weight infant. Pediatrics 2001;107.2:293-8.

17. Manzoni P, Farina D, Leonessa M, d'Oulx EA, Galletto P, Mostert M, Gomirato G. Risk factors for progression to invasive fungal infection in preterm neonates with fungal colonization. Pediatrics 2006;118.6:2359-64.

18. Singhi S, Rao DSR, Chakrabarti A. Candida colonization and candidemia in a pediatric intensive care unit. Pediatr Crit Care Med 2008; 9(1), 91-95.

19. Carey AJ, Saiman L, Polin RA. Hospital-acquired infections in the NICU: epidemiology for the new millennium. Clin Perinatol 2008;35.1:223-49.

20. Kaufman D, Boyle R, Hazen KC, Patrie JT, Robinson M, Donowitz LG. Fluconazole prophylaxis against fungal colonization and infection in preterm infants. N Engl J Med 2001;345.23:1660-6. 
21. Marr KA, Seidel K, White TC, Bowden RA. Candidemia in allogeneic blood and marrow transplant recipients: evolution of risk factors after the adoption of prophylactic fluconazole. J Infect Dis 2000;181.1:309-16.

22. Vardakas KZ, Michalopoulos A, Falagas ME. Fluconazole versus itraconazole for antifungal prophylaxis in neutropenic patients with haematological malignancies: a meta-analysis of randomised-controlled trials. Br J Haematol 2005;131.1:22-8. 